\title{
ELETROQUÍMICA E CARBONO ORGÂNICO DE UM CAMBISSOLO HÚMICO NO PLANALTO CATARINENSE SOB SISTEMA AGROFLORESTAL
}

\section{Electrochemistry and organic carbon of a Humic Inceptisol in the Santa Catarina Plateau under an agroforestry system}

\author{
Jânio dos Santos Barbosa ${ }^{*}$; Carla Eloize Carducci²; Letícia Salvi Kohn ${ }^{3}$; Kristem do Carmo Rosa Silva ${ }^{3}$ Érika \\ Andressa da Silva ${ }^{4}$; Karine Louise dos Santos ${ }^{2}$. \\ ${ }^{1 *}$ Acadêmico de Engenharia Florestal; Universidade Federal de Santa Catarina, UFSC - Campus de Curitibanos; \\ janio.jsb@gmail.com \\ 2Profa. Dra.; Universidade Federal de Santa Catarina, UFSC - Campus de Curitibanos; ec.carducci@ufsc.br, \\ karine.santos@ufsc.br \\ ${ }^{3}$ Acadêmica do curso de Agronomia; Universidade Federal de Santa Catarina, UFSC - Campus de Curitibanos; \\ leticiakohn@yahoo.com.br, kristemsilva@gmail.com \\ ${ }^{4}$ Discente do Curso de Doutorado; Dept ${ }^{\circ}$ de Ciência do Solo; Universidade Federal de Lavras, DCS/UFLA; \\ andressaerikasilva@gmail.com
}

Artigo enviado em 10/04/2016, aceito em 04/10/2016 e publicado em 20/12/2016.

\begin{abstract}
Resumo - Cambissolos são solos pouco desenvolvidos e caracterizados, de modo geral, como muito rasos, distróficos e com limitações físicas ao uso agrícola. Os sistemas agroflorestais (SAFs) surgem como uma alternativa de produção agrícola, que preserva a estrutura e a qualidade do solo. O objetivo do estudo foi avaliar o efeito da utilização do sistema agroflorestal, sobre as características eletroquímicas de um Cambissolo Húmico, na região do Planalto Catarinense. A área experimental de 0,8 ha conduzida em sistema agroflorestal na cidade de Curitibanos-SC tem como tratamentos: SAF- Erva-mate (SE), SAF- Frutífera (SF) e SAF- Agrícola (SA), sendo todos implantados em outubro de 2013 além de uma área de vegetação nativa (VN) considerada como testemunha. Foram abertas trincheiras aleatórias em cada tratamento para a coleta de amostras de solo com estrutura alterada nas camadas de 0,0-0,05 m e 0,05-0,20 m no primeiro semestre de 2014 e de 2015 para determinação dos atributos eletroquímicos: balanço de carga $(\Delta \mathrm{pH})$, ponto de carga zero (PCZ), carbono orgânico (CO) e matéria orgânica total (MO). Por meio do $\Delta \mathrm{pH}$ verificou-se que o Cambissolo Húmico é eletronegativo, a superfície dos coloides apresentou carga líquida negativa, bem como o baixo valor de PCZ, devido a grande quantidade de matéria orgânica $\left(\approx 70 \mathrm{~g} \mathrm{~kg}^{-1}\right)$. A diversidade de espécies vegetais presente no sistema agroflorestal manteve as características químicas intrínsecas do solo em estudo, alto conteúdo de matéria orgânica, eletronegatividade dos coloides organominerais e PCZ, porém contribuíram com a redução de acidez ativa logo no primeiro ano no sistema agrícola (SA) e erva-mate (SE).
\end{abstract}

Palavras-Chave - matéria orgânica, balanço de cargas, qualidade química.

\begin{abstract}
Inceptisols are undeveloped and characterized soil, generally, as very shallow, dystrophics and with physical limitations to agricultural use. Agroforestry systems (AFS) appear as an alternative agricultural production, which preserves the structure and soil quality. The aim of this study was to evaluate the effect of using agroforestry system on the electrochemical characteristics of a Inceptisol in the mountainous plateau region of Santa Catarina. The experimental area of 0.8 ha conducted in agroforestry system in the city of Curitibanos-SC. Has as treatments: SIFmate herb (SE), SIF-fruit (SF), and SIF-agriculture (SA), all beying deployed in October 2013, plus there is an area of natural vegetation $(\mathrm{VN})$ considered as a witness. Random trenches were dug in each treatment to collect soil samples with altered structure in the depths $0,0-0,05 \mathrm{~m}$ and $0,05-0,20 \mathrm{~m}$ in the first half of 2014 and 2015 for determining the electrochemical properties: charge balancing $(\Delta \mathrm{pH})$, zero charge point $(\mathrm{PZC})$, organic carbon $(\mathrm{CO})$ and total organic matter $(\mathrm{OM})$. Through the $\Delta \mathrm{pH}$ it was found that the Inceptisol is electronegative, the surface of colloids showed
\end{abstract}


negative charge, and low value of PCZ due to the large amount of organic matter $\left(\approx 70 \mathrm{~g} \mathrm{~kg}^{-1}\right)$. The diversity of plant species in agroforestry system kept the intrinsic chemical properties of the soil study, high content of organic matter, electronegativity of organo colloids and PCZ, but they contributed to the reduction of active acidity in the first year in the SIF-agriculture (SA) and SIF mate herb (SE).

Keywords - organic matter, charge balance, chemical quality.

\section{INTRODUÇÃO}

Em sistemas agroflorestais ( $\mathrm{SAFs}$ ), a prática integrada de culturas agrícolas com espécies florestais, tem se tornado uma alternativa de produção agrícola viável para agricultura, por promover melhorias na qualidade física e química do solo devido à minimização dos efeitos negativos na estrutura do solo, provocados por sistemas de manejo convencionais.

As espécies geralmente, utilizadas apresentam diferentes ciclos, portes e funções que proporcionam ao ambiente edáfico um aumento na biodiversidade, o que promove melhorias físico-químicas, hídricas e microbiológicas do solo (SILVA et al., 2011).

Os Cambissolos, solo de ocorrência na região do estudo, são caracterizados como pouco desenvolvidos, rasos, distróficos e amarelados. Segundo Pereira et al. (2010), são solos pouco estudados, devidas suas condições desfavoráveis a produção agrícola, tanto os fatores relacionados à qualidade física (elevados teores de silte) quanto a mineralogia da fração argila (predomina caulinita - formação da estrutura em blocos), estabelecendo influência direta nas características morfológicas (muito duros quando secos) a exemplo, da restrição à infiltração de água e lixiviação ao longo do perfil.

Em estudos quanto às modificações em diferentes ecossistemas deve-se avaliar a estreita relação entre a vegetação e o solo, sendo que primeiramente existem modificações nos processos físico-químicos, seja diretamente pelo suprimento de matéria orgânica, onde se apresenta cargas de superfície que contribuem para o aumento da capacidade de troca de cátions, ou indiretamente, na estruturação por meio do balanço de cargas, capacidade de retenção de cátions e fornecimento de nutrientes (ZANDONADI et al., 2014).

O objetivo do presente estudo foi avaliar o efeito da utilização do sistema agroflorestal, sobre as características eletroquímicas de um Cambissolo Húmico, na região do Planalto Serrano de Santa Catarina.

\section{MATERIAL E MÉTODOS Descrição da área experimental}

O estudo foi conduzido na área experimental agroflorestal com 0,80 ha, localizada na Universidade Federal de Santa Catarina (UFSC) na cidade de

Curitibanos, SC, implantado em outubro de 2013, sendo avaliados no primeiro semestre de 2014 e 2015. O clima da região é do tipo subtropical úmido mesotérmico (Cfb) segundo a classificação de Köppen (WREGE et al., 2011). No período da avaliação do experimento foram observadas maiores temperaturas e precipitações na região devido ao fenômeno "El Niño" (Figura 1), obtidos da estação meteorológicas da UFSC.

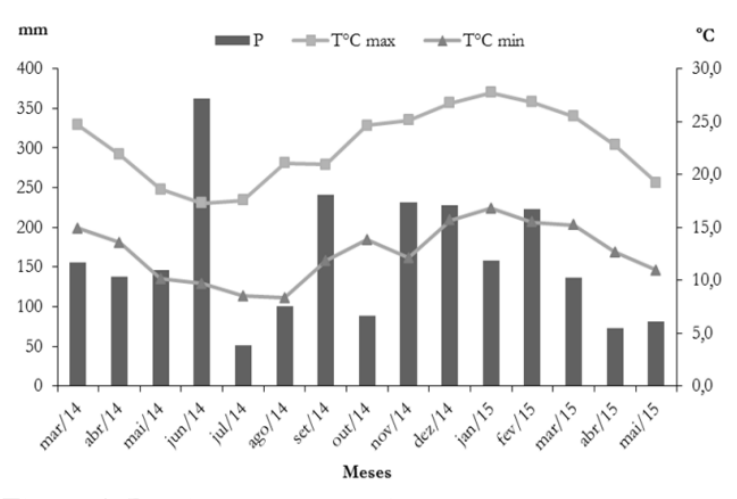

Figura 1. Precipitação mensal e temperaturas máximas e mínimas referentes ao período de 2014 e 2015 em Curitibanos, SC.

O sistema agroflorestal (SAF) está constituído em uma área onde anteriormente houve cultivo de pinus (0,40 ha), o qual foi colhido no ano de 2010. Espécies florestais nativas que já existiam no local foram deixadas para composição do SAF a exemplo, da Pinheiro-doParaná (Araucaria Angustifolia), Bracatinga (Mimosa scabrella), Fumo bravo (Solanum mauritianum), Bugreiro (Litbraea brasiliensis M.), Camboatá branco (Matayba eleagnoides), Canela (Cinnamomum verum) e Aroeiras (Lithraea molleoides). Estas espécies foram combinadas com as culturas anuais e perenes.

As áreas foram organizadas em parcelas de 500 $\mathrm{m}^{2}$ sendo essas enriquecidas por espécies de interesse econômico, compondo os seguintes tratamentos: SAF- 
Erva-mate (SE) (Ilex paraguariensis), SAF- Frutíferas (SF) utilizada a pitangueira (Eugenia uniflora L.), a goiaba serrana (Acca sellowiana), araça (Psidium cattlelyanum), ingá (Inga spp), cerejeira (Eugenia involucrata), guabiroba (Campomanesia xanthocarpa), SAF- Agrícola (SA) com rotações de culturas: milho (Zea Maiz), e mandioca (Manihot esculenta) no verão e espécies de cobertura como azevém (Lollium multiflorum), aveia (Avena sativa) e trevo (Trifolium repens) durante o inverno, o experimento consta ainda de uma área de proteção ambiental, que foi utilizada como a área testemunha - vegetação nativa (VN). Cada tratamento apresentou três repetições.

Todas as atividades de manejo do SAF evitam o revolvimento excessivo do solo (tratos culturais manuais), não utilizando defensivos agrícolas convencionais ou fertilizantes de alta solubilidade. A correção do solo foi realizada com calcário dolomítico ( $\mathrm{CaO} 28 \%, \mathrm{MgO} 18,5 \%$, PRNT 75,2\%) na área agrícola e nas covas das frutíferas $\left(6 \mathrm{Mg} \mathrm{ha}^{-1}\right)$, aplicação de cinza de caldeiras proveniente da produção de energia para os produtos florestais $\left(4 \mathrm{Mg} \mathrm{ha}^{-1}\right)$ para correção do $\mathrm{pH}$ em água ( $\mathrm{pH} 4)$ nas parcelas, fosfato natural $\left(4 \mathrm{Mg} \mathrm{ha}^{-1}[00-\right.$ 14-00 NPK] e composto orgânico como fontes de nutrientes oriundo da atividade de compostagem de resíduos vegetais (Tabela 1). Essas atividades foram realizadas nos anos de 2014 e 2015 . O único tratamento fitossanitário realizado foi o controle de formigas com o uso de iscas a base de óleo de Neen.

O solo sob o sistema agroflorestal foi classificado de acordo com a Embrapa (2013) como Cambissolo Húmico Alumínico de textura muito argilosa (Tabela 2). As análises granulométricas foram realizadas pelo emprego de agitação rápida (10.000 rpm) da suspensão do solo, contendo $\mathrm{NaOH} 1 \mathrm{M}$ (EMBRAPA, 2011).

Foram abertas três trincheiras aleatórias em cada tratamento e coletadas amostras com estrutura deformada, nas camadas de 0,0 a $0,05 \mathrm{~m}$ e 0,05 a $0,20 \mathrm{~m}$, essas profundidades foram selecionadas mediante a análise do perfil cultural o qual identifica alterações estruturais do solo promovidas pelos sistemas de manejo (TAVARES FILHO; TESSIER, 2010).

Foram determinados o $\mathrm{pH}$ em $\mathrm{H}_{2} \mathrm{O}$ e o pH em $\mathrm{KCl}$ (cloreto de potássio) $1 \mathrm{~mol} \mathrm{~L}^{-1}$, na relação solo: água ou solo: solução igual a 1:2,5 obtida pela adição de $25 \mathrm{~mL}$

Tabela 1. Atributos químicos do Cambissolo Húmico para camada 0-0,20 $\mathrm{m}$ e das cinzas de caldeiras obtidas dos produtos florestais.

\begin{tabular}{|c|c|c|c|c|c|c|c|c|c|}
\hline \multirow{2}{*}{$\begin{array}{l}\text { Atributos } \\
\text { químicos }\end{array}$} & $\mathrm{pH}$ & $\mathrm{Al}$ & $\mathrm{Ca}$ & $\mathrm{Mg}$ & $\mathrm{H}+\mathrm{Al}$ & $\mathrm{T}$ & $\mathrm{T}$ & \multirow{2}{*}{$\frac{\mathrm{K}}{\mathrm{mg} / \mathrm{dm}^{3}}$} & \multirow{2}{*}{$\begin{array}{c}\mathrm{MO} \\
\%\end{array}$} \\
\hline & $\mathrm{H}_{2} \mathrm{O}$ & \multicolumn{6}{|c|}{ 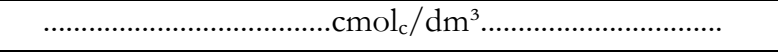 } & & \\
\hline Bloco 01 & 3,54 & 6,5 & 0,66 & 0,53 & 38,4 & 40 & 7,86 & 68 & 5,9 \\
\hline Bloco 02 & 3,75 & 575 & 0,9 & 0,66 & 34,45 & 36,17 & 7,47 & 61 & 6,4 \\
\hline Bloco 03 & 3,75 & 5,5 & 0,63 & 0,51 & 32,9 & 34,13 & 6,73 & 37 & 5,5 \\
\hline Testemunha & 4,19 & 4,5 & 1,18 & 0,66 & 27,7 & 29,63 & 6,43 & 54 & 4,2 \\
\hline \multirow{2}{*}{ Cinzas } & Moc & $\mathrm{Ct}$ & Co & Rms & $\mathrm{P}$ & $\mathrm{Ca}$ & K & $\mathrm{Mg}$ & $\mathrm{S}$ \\
\hline & \multicolumn{9}{|c|}{$\%$} \\
\hline Umidade & 7,00 & 4,87 & 3,89 & 20,92 & 1,08 & 1,56 & 2,11 & 0,53 & 0,18 \\
\hline
\end{tabular}

Bloco 1: SAF Frutífera e Erva-mate; Bloco 02: SAF Erva-mate e Agrícola; Bloco 03: SAF Agrícola e Frutífera; Testemunha: vegetação nativa; T: capacidade de troca catiônica (CTC) pH 7,00, t: capacidade de troca catiônica (CTC) efetiva, MO: matéria orgânica; Moc: matéria orgânica compostável, Ct: carbono total, Co: carbono orgânico, Rms: resíduo mineral solúvel. Em cada Bloco está inserida uma repetição de cada tratamento. 
Tabela 2. Caracterização granulométrica do Cambissolo Húmico em diferentes camadas em Sistema Agroflorestal no Planalto Catarinense.

\begin{tabular}{|c|c|c|c|c|c|c|}
\hline \multirow{3}{*}{ Tratamentos } & \multicolumn{3}{|c|}{$0-0,05 \mathrm{~m}$} & \multicolumn{3}{|c|}{$0,05-0,20 \mathrm{~m}$} \\
\hline & Argila & Areia & Silte & Argila & Areia & Silte \\
\hline & \multicolumn{6}{|c|}{ 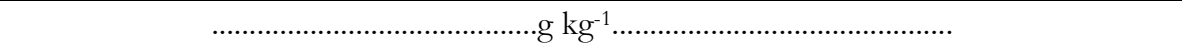 } \\
\hline VN & 598 & 91 & 311 & 645 & 88 & 267 \\
\hline SA & 648 & 61 & 291 & 623 & 62 & 315 \\
\hline SF & 655 & 67 & 278 & 640 & 58 & 302 \\
\hline SE & 656 & 76 & 268 & 578 & 78 & 345 \\
\hline
\end{tabular}

SE: SAF- Erva-mate, SA: SAF- Agrícola, SF: SAF- Frutífera e VN: vegetação nativa.

de água ou de solução a $10 \mathrm{~cm}^{3}$ de solo, seguida de agitação para completa homogeneização e formação da suspensão. Após 60 minutos, foi determinado o pH das suspensões, respectivamente, avaliaram a acidez ativa $\left(\mathrm{pH}_{\mathrm{H} 2 \mathrm{O}}\right)$ e a acidez trocável $\left(\mathrm{pH}_{\mathrm{KCL}}\right)$ (EMBRAPA, 2011). A partir desses valores foi realizado o balanço de cargas $(\Delta \mathrm{pH})$ de acordo com a equação indicada por Uehara e Gillman (1980a,b):

$$
\Delta \mathrm{pH}=p H_{K C l}-p H_{H 2 O}
$$

Ainda foi estimado o ponto de carga zero (PCZ), conforme a equação proposta por Uehara e Gillman, (1980a):

$$
\mathrm{PCZ}=2 p H_{K C l}-p H_{H 2 O}
$$

Seguindo a metodologia da Embrapa (2011) foi determinado o valor de carbono orgânico do solo, sendo esses valores transformados para matéria orgânica total (MO) de acordo com a equação: $\mathrm{MO}=$ $\mathrm{C} * 1,724$, onde: $\mathrm{MO}=$ matéria orgânica $\left(\mathrm{g} \mathrm{kg}^{-1}\right) ; \mathrm{C}=$ é o carbono orgânico $\left(\mathrm{g} \mathrm{kg}^{-1}\right)$ e 1,724 é um fator utilizado em virtude de se admitir que, na composição média do húmus, o carbono participa com $58 \%$, sendo o valor de $\mathrm{MO}$ obtido pelo método da oxidação por dicromato de potássio e a titulação com sulfato ferroso (método Walkley \& Black).

Os resultados foram submetidos à análise da variância e, quando significativa, as médias foram comparadas pelo teste de Tukey $(p<0,05)$, por meio do programa computacional SISVAR (FERREIRA, 2011).

\section{RESULTADO E DISCUSSÃO}

Observaram-se diferenças significativas entre os tratamentos testados ao longo do tempo (Figura 2). A acidez ativa do solo determinada pelo $\mathrm{pH}_{\mathrm{H} 2 \mathrm{O}}$ diminuiu de 2014 para 2015 em todos os tratamentos, onde foi possível verificar que o SAF- Erva-mate (SE) obteve o maior valor de $\mathrm{pH}$ na camada superficial do solo, passando de 4,31 no primeiro ano para 5,42 no ano de 2015 igualando-se ao SAF- Agrícola (SA). Baixos valores de $\mathrm{pH}$ são característica de solos ácidos, mas é notável a diminuição nos valores de acidez ativa devido a utilização dos corretivos como: calagem e cinzas de caldeiras, este último ainda apresenta potencialidades de utilização na fertilização de plantas (macro e micronutrientes) e como corretivo da acidez do solo, além de ser uma alternativa de baixo custo (BONFIM-SILVA et al., 2011). 

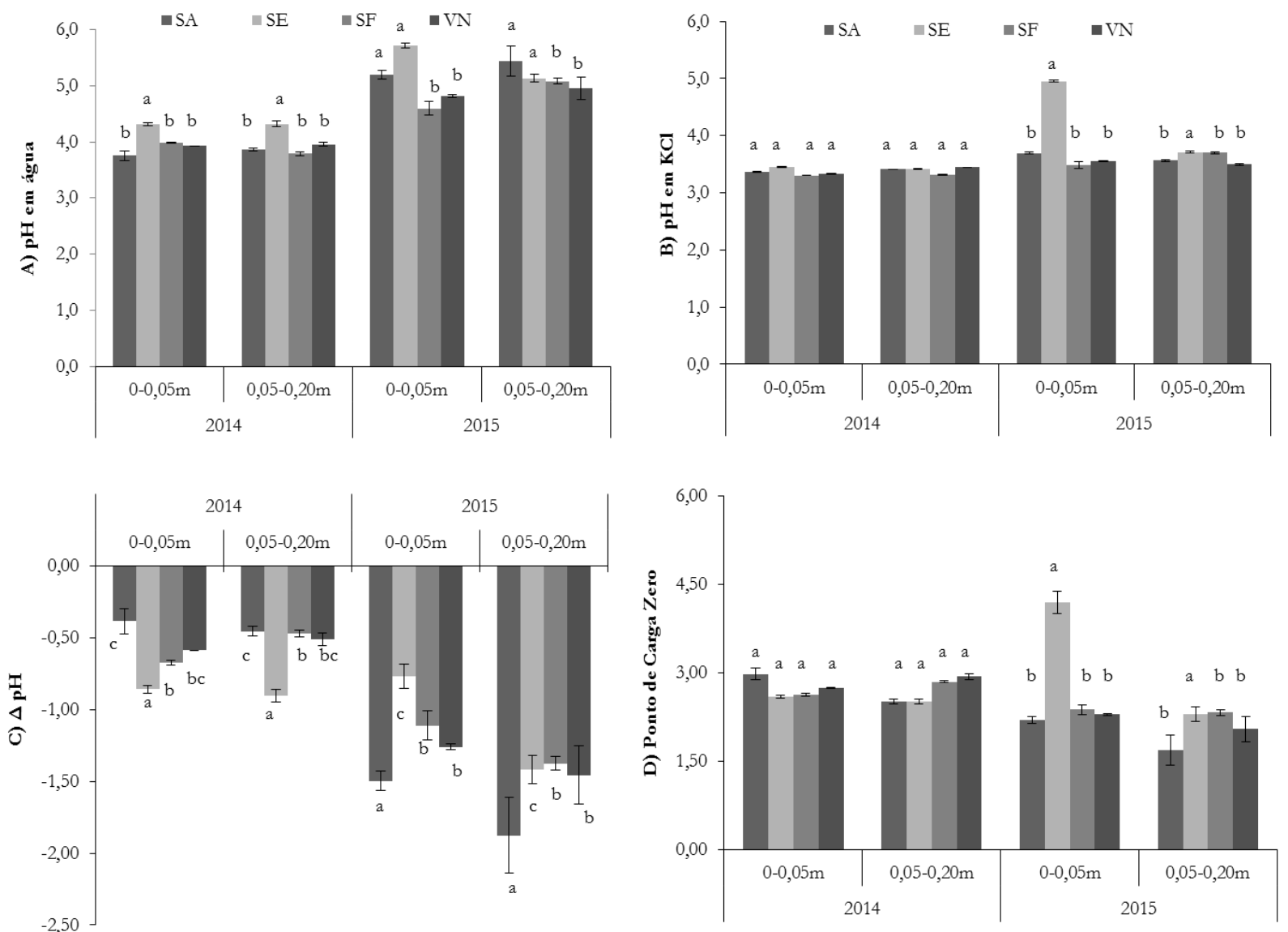

Figura 2. A) $\mathrm{pH}$ em água, B) $\mathrm{pH}$ em $\mathrm{KCl}, \mathrm{C})$ balanço de carga líquida $(\Delta \mathrm{pH}) \mathrm{D}) \mathrm{PCZ}$ - ponto de carga zero para os anos de 2014 e 2015, nas camadas de 0-0,05 m e 0,05-0,20 m do Cambissolo Húmico sob SAF no Planalto Catarinense. Médias seguidas de letras minúsculas não diferem entre si teste de Tukey $(\mathrm{p}<0,05)$ no tempo dentro das camadas e tratamentos. As barras de erro representam o erro padrão da média.

Em geral, os valores de $\mathrm{pH}_{\mathrm{H} 2 \mathrm{O}}$ foram os maiores comparados aos de $\mathrm{pH}_{\mathrm{KCl}}$ (Figura 2 B). Esses resultados são conferidos ao efeito da solução de $\mathrm{KCl}$, que, em contato com a amostra de solo, induz a troca de cátions devido à maior concentração dos íons trocáveis $\left(\mathrm{K}^{+}\right)$, liberando íons $\mathrm{H}^{+}$e $\mathrm{Al}^{3+}$ para a solução, com consequente aumento da acidez (EBELING et al., 2008).

Para $\mathrm{pH}_{\mathrm{KCl}}$ (acidez trocável) no primeiro ano não houve diferenças significativas entre os tratamentos, mas para 2015 é notório o valor de $\mathrm{pH}$ 4,3 para o SE pois se sobressaiu em relação aos demais valores. Em um estudo realizado por Favero et al. (2008), diz que a redução da acidez trocável em solo sob um sistema agroflorestal pode estar relacionada ao maior incremento em matéria orgânica nesse solo, o que pode complexar o alumínio trocável $\left(\mathrm{Al}^{3+}\right)$ com formas mais lábeis de matéria orgânica.
O balanço de cargas $(\Delta \mathrm{pH})$ (Figura $2 \mathrm{C}$ ) atribuído pela diferença do $\mathrm{pH}_{\mathrm{KCl}}$ e $\mathrm{pH}_{\mathrm{H} 2 \mathrm{O}}$ resultou em carga líquida negativa na superfície dos coloides, para os dois anos com diferenças significativas para ambos. Os maiores valores foram SE -0,88 e SA -1,69 para 2014 e 2015, respectivamente. O que se deve em grande parte a presença de matéria orgânica e caulinita, pois estes compostos apresentam baixo ponto de carga zero (PCZ), ou seja, o Cambissolo Húmico em estudo se caracteriza quimicamente por ser um solo composto por coloides eletronegativo. Vale ressaltar o alto conteúdo de argila desse solo (Tabela 2) o que contribui positivamente com o aumento da capacidade de retenção de cátions e água além de estabilizar os agregados (FONTES et al., 2001; VITORINO et al., 2003).

Um valor de carga líquida positiva subsuperficial $(0,05-0,20 \mathrm{~m})$ pode ser desejável em 
algumas situações por se reter ânions $\left(\mathrm{NO}_{3}^{-}, \mathrm{HPO}^{-}\right.$, $\mathrm{H}_{2} \mathrm{PO}_{4}^{-}, \mathrm{SO}_{4}^{-}, \mathrm{HMoO}_{4}^{-}$), contribuindo para uma melhor nutrição das plantas. No entanto, pode ter efeitos drásticos quando se trata de adsorção específica como, a que ocorre para os fosfatos em solos oxídicos (Latossolos). Fontes et al. (2001) citam que em horizontes superficiais valores de carga líquida negativa são altamente desejáveis para que se tenha uma boa retenção de cátions.

Tendo-se em mente que as cargas superficiais dos coloides desempenham um papel muito importante nas reações de troca que ocorrem nos solos, foi determinado o PCZ (Figura 2 D) para se ter uma referência da carga líquida da superfície dos coloides organominerais para um determinado valor de pH (COSTA et al., 2009), o PCZ foi significativo no segundo ano com médias variando de 1,7 a 4,2 para SA e SE, respectivamente, corroborando com o balanço de cargas $(\Delta \mathrm{pH})$, onde esses valores são típico da matéria orgânica do solo.

A matéria orgânica do solo (MO) apresentou um alto conteúdo, o que de acordo com Embrapa (2013) o caracteriza o horizonte húmico deste solo, principalmente na camada $0-0,05 \mathrm{~m}$, devido ao constante aporte de resíduo vegetal proporcionado pelo sistema agroflorestal (Tabela 3).

As maiores médias nas duas camadas ocorreram para o ano de 2014, porém não houve

Tabela 3. Valores de Matéria orgânica do Cambissolo Húmico nas camadas de 0-0,05 m e 0,05-0,20 m nos anos de 2014 e 2015 em Sistema Agroflorestal no Planalto Catarinense.

\begin{tabular}{|c|c|c|c|c|}
\hline \multirow{2}{*}{$\begin{array}{l}\text { Atributo químico } \\
\text { Ano }\end{array}$} & \multicolumn{2}{|c|}{$\begin{array}{l}0-0,05 \mathrm{~m} \\
\mathrm{MO}\end{array}$} & \multicolumn{2}{|c|}{$\begin{array}{c}0,05-0,20 \mathrm{~m} \\
\mathrm{MO}\end{array}$} \\
\hline & 2014 & 2015 & 2014 & 2015 \\
\hline Tratamentos & & ....................... & ............ & \\
\hline SA & $76,98 \mathrm{aA}$ & $67,73 \mathrm{aB}$ & $78,49 \mathrm{bA}$ & $52,9 \mathrm{bB}$ \\
\hline SE & $82,25 \mathrm{aA}$ & $69,70 \mathrm{aB}$ & $72,72 \mathrm{bA}$ & $52,86 \mathrm{bB}$ \\
\hline SF & $72,47 \mathrm{aA}$ & $62,79 \mathrm{aB}$ & $58,18 \mathrm{bA}$ & $58,51 \mathrm{bB}$ \\
\hline $\mathrm{VN}$ & $80,75 \mathrm{aA}$ & $70,07 \mathrm{aB}$ & $69,46 \mathrm{bA}$ & $59,72 \mathrm{bB}$ \\
\hline
\end{tabular}

SE: SAF- Erva-mate, SA: SAF- Agrícola, SF: SAF- Frutíferas e VN: vegetação nativa. MO: matéria orgânica. Médias de tratamentos em cada camada, seguidas de letras minúsculas iguais na coluna e médias de tratamentos seguidas de letras maiúsculas iguais na linha não diferem pelo teste de Tukey $(\mathrm{p}<0,05)$.

diferença significativa entre os tratamentos. Ao avaliar a evolução do conteúdo de MO de um ano para outro de implantação houve significativa redução da MO nas duas camadas, cerca de $70 \mathrm{~g} \mathrm{~kg}^{-1}$ para $55 \mathrm{~g} \mathrm{~kg}^{-1} \mathrm{em} 2015$ na camada subsuperficial, isso pode estar associado as intervenções realizadas devido a semeadura e plantio

de novas culturas associada as alterações climáticas ocorridos no ano de 2015 que foi atípico na região com variações constantes, ou seja, elevação da temperatura e intensidade pluviométrica, devido ao fenômeno climático 'El Niño', logo, pode ter ocasionado a rápida decomposição da matéria orgânica e acelerado as reações químicas do solo (Costa et al., 2006; LOSS et al., 2009).

Entretanto, vale ressaltar que mesmo com a redução de $\mathrm{MO}$ de um ano para outro, esses valores ainda são altos e corroboram com os dados de MO encontrados por Silva et al. (2013) ao avaliarem um sistema conservacionista do solo para o cultivo de cafeeiros. Sistemas de manejo do solo que empregam práticas de conservação, como o SAF, promovem a melhoria ou manutenção dos atributos físicos, químicos e biológicos benéficos ao solo e, o que promove a sustentabilidade do meio agrícola (LOSS et al., 2009; SILVA et al., 2013).

Ressalta-se que a matéria orgânica é um componente cimentante das partículas minerais do solo, com ação direta na estabilização da estrutura do solo e, dessa forma atua no equilíbrio dos processos dinâmicos que envolvem o fluxo de ar e água no sistema solo. A matéria orgânica ainda contribui para a complexação de elementos químicos catiônicos do solo, tanto micronutrientes como elementos considerados tóxicos $\left(\mathrm{Al}^{3+}\right)$ pela elevação da capacidade do solo em reter cátions (SALTON et al., 2008; LOSS et al., 2009; SILVA et al., 2013).

\section{CONCLUSÃO}

Por meio do $\Delta \mathrm{pH}$ verificou-se que o Cambissolo Húmico apresentou coloides com carga líquida negativa, bem como baixo valor de PCZ, 
devido a grande quantidade de matéria orgânica $(\approx 70$ $\left.\mathrm{g} \mathrm{kg}^{-1}\right)$. A diversidade de espécies vegetais presente no sistema agroflorestal manteve as características químicas intrínsecas do solo em estudo, alto conteúdo de matéria orgânica, eletronegatividade dos coloides organominerais e baixo PCZ, porém contribuíram com a redução de acidez ativa logo no primeiro ano de condução do sistema.

\section{REFERÊNCIAS}

BONFIM-SILVA, E. M.; SILVA, T. J. A.; CABRAL, C. E. A.; KROTH, B. E.; REZENDE, D. Desenvolvimento inicial de gramíneas submetidas ao estresse hídrico. Revista Caatinga, v. 24, n. 2, p. 180-186, 2011.

COSTA, A.; ALBUQUERQUE, J. A.; ERNANI, P. R.; BAYER, C.; MERTZ, L. M. Alterações físicas e químicas num Cambissolo Húmico de campo nativo após a correção da acidez. Revista de Ciências Agroveterinárias, v. 5, n. 2, p. 118-130, 2006.

COSTA, E. T. S.; GUILHERME, L. R. G.; CURI, N.; LOPES, G.; VISIOLI, E. L.; OLIVEIRA, L. C. A. Caracterização de subproduto da indústria de alumínio e seu uso na retenção decádmio e chumbo em sistemas monoelementares. Quimica Nova, v. 32, n.4, p. 868-874, 2009.

EBELING, A. G.; ANJOS, L. H. C.; PÉREZ, D. V.; PEREIRA, M.G.; VALLADARES, G. S. Relação entre acidez e outros atributos químicos em solos com teores elevados de matéria orgânica. Bragantia, v. 67, n. 2, p. 261-266, 2008.

EMBRAPA - Empresa Brasileira de Pesquisa Agropecuária. Manual de Métodos de Análises do solo. Embrapa solos, 2011.

EMBRAPA - Empresa Brasileira de Pesquisa Agropecuária. Solos. Sistema brasileiro de classificação de solos. ed. Rio de Janeiro: Embrapa Solos, 2013.

FAVERO, C.; LOVO, I. C.; MENDONÇA, E. S. Recuperação de Área Degradada com Sistema Agroflorestal no Vale do Rio Doce, Minas Gerais. Revista Arvore, v. 32, n. 5, p. 861-868, 2008.

FERREIRA, D. F. Sisvar: a computer statistic alanalysis system. Ciência e Agrotecnologia, v. 35, n. 6, p.1039-1042, 2011.
FONTES, M. P. F.; CAMARGO, O. A.; SPOSITO, G. Eletroquímica das partículas coloidais e sua relação com a mineralogia de solos altamente intemperizados. Scientia Agrícola, v. 58, n. 3, p. 627-646, 2001.

LOSS, A.; PEREIRA, M.G.; SCHULTZ, N.; ANJOS, L.H.C.; SILVA, E.M.R. Atributos químicos e físicos de um Argissolo Vermelho-Amarelo em sistema integrado de produção agroecológica. Pesquisa Agropecuária Brasileira, v. 44, n. 1, p. 68-75, 2009.

PEREIRA, T. T. C.; KER, J. C.; SCHAEFER, C. E. G. R.; BARROS, N. F.; NEVES, J. C. L.; ALMEIDA, C. C. Gênese de latossolos e cambissolos desenvolvidos de rochas pelíticas do grupo Bambuí Minas Gerais. Revista Brasileira de Ciência do Solo, v. 34, n. 4, p. 1283-1295, 2010.

SALTON, J. C.; MIELNICZUK, J.; BAYER, C.; BOENI, M.; CONCEIÇÃO, P. C.; FABRICIO, A. C.; MACEDO, M. C. M.; BROCH, D. L. Agregação e estabilidade de agregados do solo em sistemas agropecuários em Mato Grosso do Sul. Revista Brasileira de Ciência do Solo, v. 32, n. 1, p. 11-21, 2008.

SILVA, E. A.; OLIVEIRA, G. C.; CARDUCCI, C. E.; SILVA, B. M.; OLIVEIRA, L. M.; COSTA, J. C. Doses crescentes de gesso agrícola, estabilidade de agregados e carbono orgânico em Latossolo do Cerrado sob Cafeicultura. Revista de Ciências Agrarias, v. 56, n. 1, p. 25-32, 2013.

SILVA, D. C.; SILVA, M. L. N.; CURI, N.; OLIVEIRA, A. H.; SOUZA, F.; Martins, S. G.; MACEDO, R. L. G. Atributos do solo em sistemas agroflorestais, cultivo convencional e floresta nativa. Revista de estudos ambientais, v. 13, n. 1, p. 77-86, 2011.

TAVARES FILHO, J.; TESSIER, D. Effects of different management systems on porosity of oxisols in Paraná, Brasil. Revista Brasileira de Ciência do Solo, v. 34, n. 3, p. 899-906, 2010.

UEHARA, G.; GILLMAN, G. P. Charge characteristics of soils with variable and permanent charge minerals: I. Theory. Soil Science Society of America Journal, v. 44, n. 2, p. 250-252, 1980 a.

UEHARA, G.; GILLMAN, G. P. Charge characteristics of soils with variable and permanent charge minerals: II. Experimental. Soil Science Society of America Journal, v. 44, n. 2, p. 252-255, 1980 b. 
VITORINO, A. C. T.; FERREIRA, M. M.; CURI, N.; LIMA, J. M.; SILVA, M. L. N.; MOTTA, P. E. F. Mineralogia, química e estabilidade de agregados do tamanho de silte de solos da Região Sudeste do Brasil. Pesquisa Agropecuária Brasileira, v. 38, n. 1, p. 133-141, 2003.

ZANDONADI, D. B.; SANTOS, M. P.; MEDICI, L. O.; SILVA, J. Ação da matéria orgânica e suas frações sobre a fisiologia de hortaliças. Horticultura Brasileira, v. 32, n. 1, p. 14-20, 2014.

WREGE, M. S.; STEINMETZ, S.; REISSER JUNIOR, C.; ALMEIDA, I. R. Atlas climático da Região Sul do Brasil: Estados do Paraná, Santa Catarina e Rio Grande do Sul. Pelotas: Embrapa Clima Temperado; Colombo: Embrapa Florestas, 2011. 